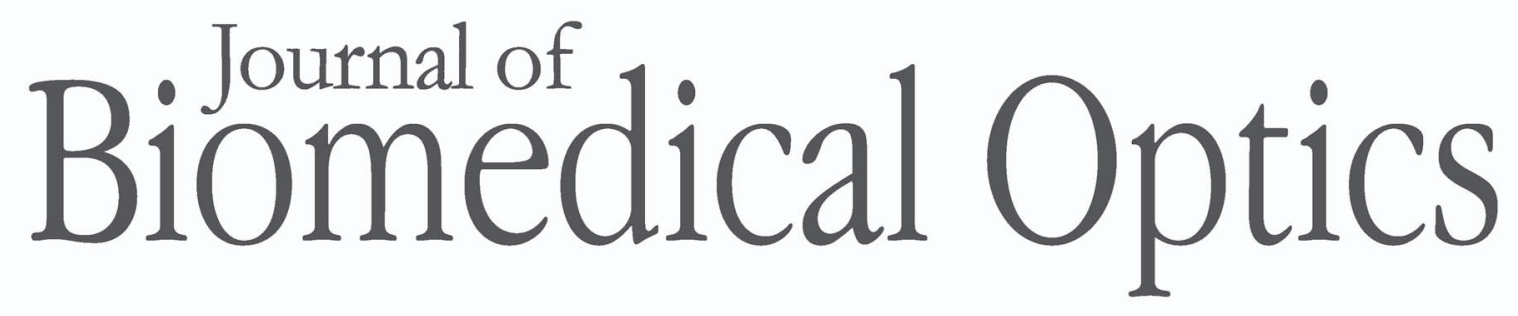

\title{
Recovery of macular pigment spectrum in vivo using hyperspectral image analysis
}

Amani A. Fawzi

Noah Lee

Jennifer H. Acton

Andrew F. Laine

R. Theodore Smith 


\title{
Recovery of macular pigment spectrum in vivo using hyperspectral image analysis
}

\author{
Amani A. Fawzi, ${ }^{a}$ Noah Lee, ${ }^{b}$ Jennifer H. Acton, ${ }^{c}$ Andrew F. Laine, ${ }^{b}$ and R. Theodore Smith ${ }^{c}$ \\ a University of Southern California, Doheny Eye Institute, Keck School of Medicine, Department of Ophthalmology, \\ 1450 San Pablo Ave, Suite 3614, Los Angeles, California 90033 \\ ${ }^{b}$ Columbia University, Heffner Biomedical Imaging Laboratory, Department of Biomedical Engineering, \\ 351 Engineering Terrace MC-8904, 1210 Amsterdam Avenue, New York, New York 10027 \\ ${ }^{c}$ Columbia University, Harkness Eye Institute, Department of Ophthalmology, 635 West 165 Street, P.O. Box 12, \\ New York, New York 10032
}

\begin{abstract}
We investigated the feasibility of a novel method for hyperspectral mapping of macular pigment (MP) in vivo. Six healthy subjects were recruited for noninvasive imaging using a snapshot hyperspectral system. The three-dimensional full spatial-spectral data cube was analyzed using non-negative matrix factorization (NMF), wherein the data was decomposed to give spectral signatures and spatial distribution, in search for the MP absorbance spectrum. The NMF was initialized with the in vitro MP spectrum and rank 4 spectral signature decomposition was used to recover the MP spectrum and optical density in vivo. The recovered MP spectra showed two peaks in the blue spectrum, characteristic of MP, giving a detailed in vivo demonstration of these absorbance peaks. The peak MP optical densities ranged from 0.08 to 0.22 (mean $0.15+/-0.05$ ) and became spatially negligible at diameters 1100 to $1760 \mu \mathrm{m}$ (4 to $6 \mathrm{deg}$ ) in the normal subjects. This objective method was able to exploit prior knowledge (the in vitro MP spectrum) in order to extract an accurate in vivo spectral analysis and full MP spatial profile, while separating the MP spectra from other ocular absorbers. Snapshot hyperspectral imaging in combination with advanced mathematical analysis provides a simple cost-effective approach for MP mapping in vivo. (C) 2011 Society of Photo-Optical Instrumentation Engineers (SPIE). [DOI: 10.1117/1.3640813]
\end{abstract}

Keywords: hyperspectral imaging; ophthalmology; image analysis.

Paper 11301R received Jun. 14, 2011; revised manuscript received Aug. 23, 2011; accepted for publication Aug. 30, 2011; published online Oct. 6, 2011.

\section{Introduction}

The role of macular pigment (MP) in ocular health and disease remains controversial. It has been suggested as a scavenger for a reactive oxygen species and a blue light filter, and, hence, to have a protective role against light-induced damage to the photoreceptor/RPE complex. ${ }^{1,2}$ Lutein (L) and zeaxanthin (Z), the carotenoids that constitute $\mathrm{MP}^{3}$ have antioxidant properties that appear to be protective against blue light. ${ }^{4}$ The optical filtering properties of MP, to reduce chromatic aberration and glare, have also been described. ${ }^{1}$ Its role in age-related macular degeneration (AMD) remains controversial. While some have proposed a preventative role, ${ }^{5,6}$ others have disputed this role in their populations of AMD. ${ }^{7,8}$ Furthermore, the ability to modify the density of macular pigment through dietary supplements remains an important, yet unanswered question. Most studies have shown a significant relationship and this has been comprehensively reviewed. ${ }^{9,10}$

MP measures are subject to large amounts of variability in normal subjects. ${ }^{11-13}$ An obstacle to clearly establishing the role of MP in retinal disease is the lack of a validated, clinically applicable, and widely accessible tool to quantify the MP in vivo. Noninvasive MP assessment methods include psychophysical techniques and objective measures. Psychophysical methods such as the matching tasks of heterochromic flicker photometry (HFP) (Refs. 12, 14-16) or minimum motion photometry ${ }^{17,18}$

Address all correspondence to: R. Theodore Smith, Columbia University, Harkness Eye Institute, 635 West 165 Street, P.O. Box 12, New York, New York 10032. Tel: 212-342-1849; Fax: 212-342-3187; E-mail: rts1@columbia.edu. assume normal retinal function and uniform lens density within the area of measurement and rely on the accuracy of patient responses. Patients with advanced ocular disease tend to experience the greatest difficulty with such tests. ${ }^{15}$ Commercially available psychophysical measures of MP generate a single value, rather than a complete profile of measures for each eccentricity. Objective methods include reflectance imaging, ${ }^{19-21}$ autofluorescence (AF) imaging, ${ }^{22-24}$ and Raman spectroscopy. ${ }^{25,26}$ Reflectance and AF imaging give the spatial distribution of MP, but in some cases require accurate fixation and require prebleaching to avoid confounding absorption by photopigments, which involves unpleasant light levels. Reflectance measures are affected by stray light, unless confocal imaging is used. The AF technique assumes that the relative spectral energy of lipofuscin fluorescence is constant across the central retina. ${ }^{3}$ Despite the high chemical specificity of Raman spectroscopy, its signal can be attenuated by lenticular absorbance or scattering and maximal pupil dilation is required for measurement. ${ }^{3,27}$

Hence, there is a need for a rapid, objective, and simple method to noninvasively evaluate the MP. In our previous research, ${ }^{28,29}$ we evaluated a hyperspectral reflectometry camera, which captures a 20 deg field with 76 bands of spectral information, in conjunction with a partially constrained unsupervised data mining approach (blind source separation) to demonstrate the spectra that colocalized with drusen. Here, we applied this technique to quantify the MP in a group of healthy eyes in vivo. Our results are comparable to measurements made with other

1083-3668/2011/16(10)/106008/9/\$25.00 @ 2011 SPIE 


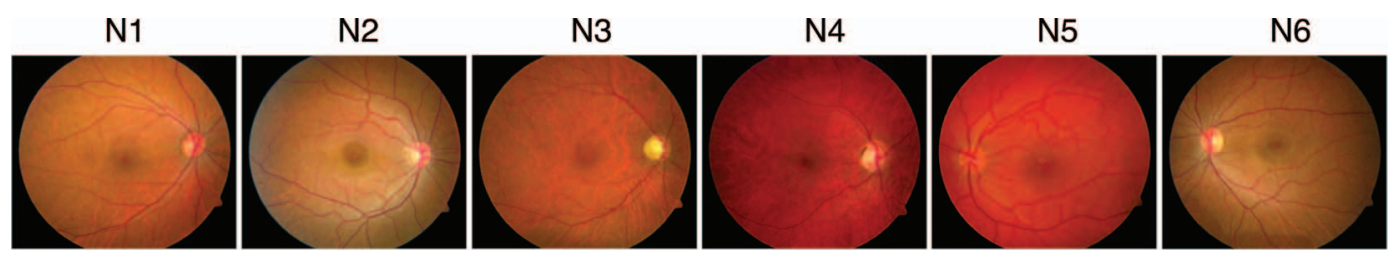

Fig. 1 Color fundus images of six healthy subjects (N1 to N6). N1, N2, and N6 show darkly pigmented individuals, while N3 is lightly pigmented (blond fundus).

reflectance approaches. There has been recent interest in hyperspectral imaging using a modified fundus camera and the various systems have been briefly reviewed. ${ }^{30}$ Prior multispectral retinal image analysis has been restricted, either in spatial extent to single line scans ${ }^{31}$ or to pointwise acquisition, ${ }^{32}$ or spectrally to a limited number of spectral bands. ${ }^{33-35}$ While excellent focal analysis of MP has been possible with two wavelength reflectance ${ }^{36,37}$ or $\mathrm{AF}^{36}$ we wanted to investigate the full 20 deg field with 76 bands of spectral information and explore the use of blind source separation to obtain detailed spectral characterization of MP in vivo.

\section{Methods}

\subsection{Subjects}

We recruited six healthy volunteers (mean age $32.8, \pm 12.1$, range 22 to 55 years, three males and three females; Fig. 1) from the University of Southern California. Each patient underwent a complete eye exam followed by pupil dilation and imaging with the hyperspectral camera. Photoreceptor pigment prebleaching was not systematically attempted.

This study was approved by the institutional review boards of the University of Southern California and Columbia University and adhered to the tenets of the Declaration of Helsinki for research involving human subjects. Each participant gave informed consent after explanation of the nature and possible consequences of the study.

\subsection{Hyperspectral Camera}

To avoid image coregistration and motion artifacts in spectral space, this camera employed a novel technique, computed tomographic imaging spectrometer (CTIS), for hyperspectral retinal imaging. The hyperspectral imaging device consists of an imaging spectrometer attached to a commercial fundus camera. This device captures a full spatial-spectral image cube (20 deg field, $186 \times 186$ pixel spatial resolution) in a single camera flash. CTIS uses diffractive optics to multiplex spatial-spectral information onto a focal plane array. With less than $20 \mathrm{msec}$ integration time, the retinal CTIS system acquired 76 contiguous spectral bands between 420 and $720 \mathrm{~nm}$ at $4 \mathrm{~nm}$ resolution ${ }^{38,39}$ (Fig. 2), thus obviating the need for subject immobilization for precise image registration. ${ }^{40}$

\subsection{Image Reconstruction}

The images were reconstructed, using the previously described approach. ${ }^{39}$ Briefly, expectation maximization algorithms are employed on the diffraction pattern to reconstruct the image cube. The reconstructed cubes were then anonymously transferred to Columbia University for further data analysis.

\subsection{Data Analysis}

We employed a data mining approach to explore and search for constituent pigment spectra of the retina. In particular, we focused on extracting the spatio-spectral MP signatures in healthy subjects. An unsupervised learning algorithm called non-negative matrix factorization (NMF) (Refs. 41 and 42) was employed with a partial constraint in the form of a physiologic meaningful initialization scheme. NMF as usually implemented decomposes the data as a linear sum of sources times abundances; specifically, NMF decomposes a multivariate dataset $\mathbf{X}$ into two matrices: a matrix of spectral signatures $\mathbf{S}$ and their corresponding spatial distribution A. NMF assumes the linear model

$$
\mathbf{X}=\mathbf{A S} \text {, subject to } \mathbf{A}>0, \mathbf{S}>0,
$$

where $\mathbf{X}$ is the hyperspectral data, $\mathbf{A}$ is the matrix of nonnegative spatial abundance images, and $\mathbf{S}$ are the constituent

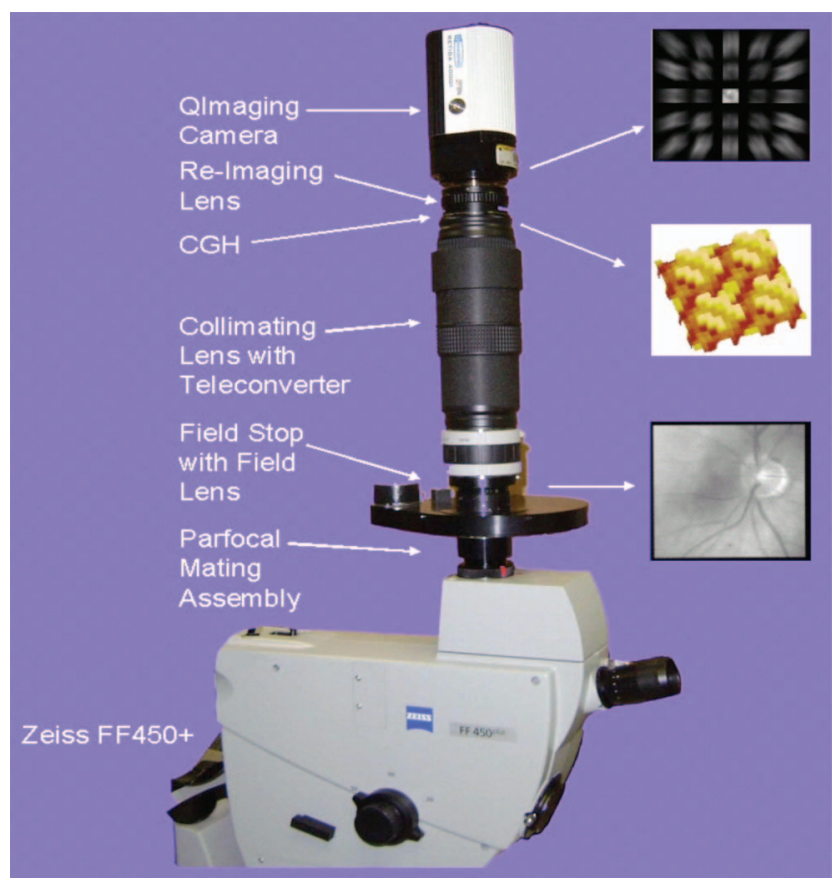

Fig. 2 Zeiss FF450 + fundus camera shown with CTIS attached. The normal $35-\mathrm{mm}$ format image from the Zeiss is field-stopped by the CTIS (insert, bottom right) and relayed using demagnification through the computer-generated holographic $(\mathrm{CGH})$ grating (insert, middle right). Diffraction patterns are shown (insert, top right). 


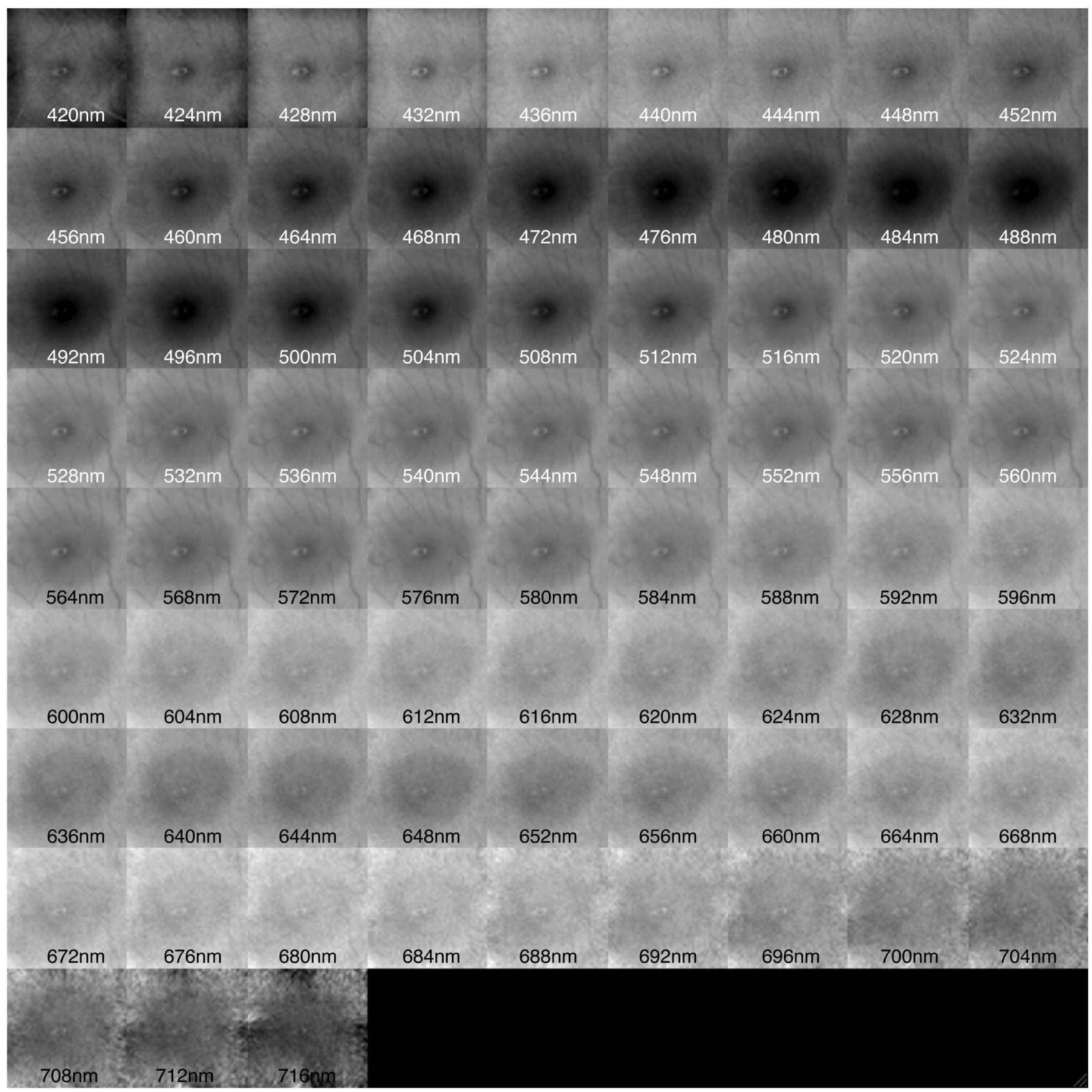

Fig. 3 Montage of a normal hyperspectral cube. The hypercube for N6 with 75 spectral bands starting from 420 to 720 nm. The montage shows postprocessed spectral bands, where the data was log10-transformed and each spectral band was normalized by its standard deviation. Negative values have been clipped and replaced with 2.2E-16. The post-processed hyperspectral cube has a range $[0,1]$. The fundus clinically was dark-pigmented.

positive spectra. ${ }^{43,44}$ Unlike principal component analysis, NMF imposes positivity constraints on the spectra and spatial abundance images, consistent with physical reality. Such constraints yield a parts-based representation of the hyperspectral cube that allows an individual signature to be obtained, i.e., its spatiospectral characterization, which has a physically meaningful interpretation.

We used NMF in a modified format on the hyperspectral reflectance data cube to recover and investigate absorbance signatures. To do this, the spectral reflectance $R$ can be written with the following simplified model (adapted from
Refs. 20 and 45):

$$
R=R_{\mathrm{SC}} \times 10^{(-2 D)},
$$

where $R_{\mathrm{SC}}$ is the reflectance of the sclera and $D$ is the total optical density (OD) of all absorbers. To convert from reflectance to absorbance the data is $\log$-transformed, assuming $D$ to be $D$ $=\mathrm{OD}_{\mathrm{MP}}+\mathrm{OD}_{\mathrm{SubT}}$, where MP denotes macular pigment and SubT is the subtotal of all optical densities of all absorbers except MP. For $\log _{10}$ of the data for each wavelength, we get

$$
-\log _{10}(R)=-\log _{10}\left(R_{\mathrm{SC}}\right)+2 \times \mathrm{OD}_{\mathrm{MP}}+2 \times \mathrm{OD}_{\mathrm{SubT}} .
$$




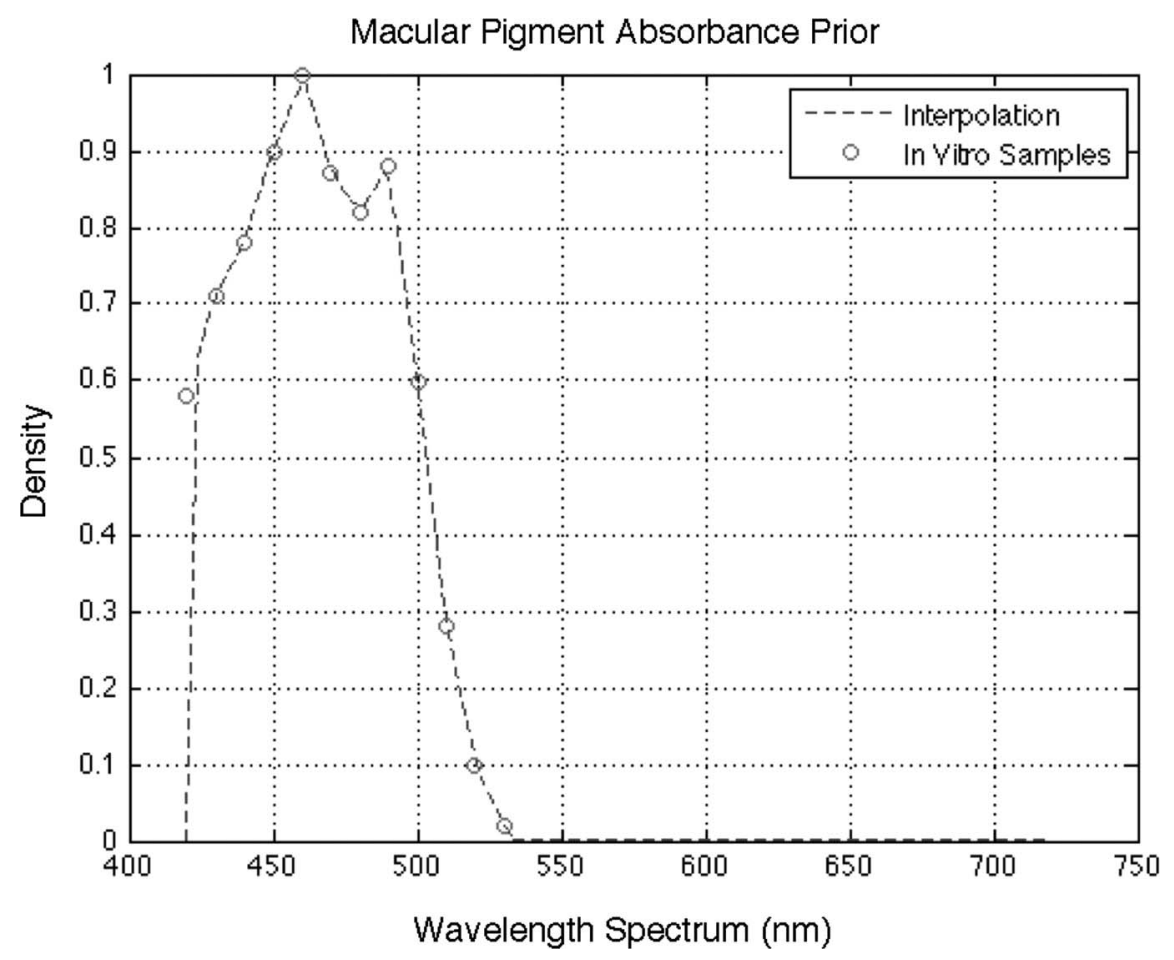

Fig. $4 \mathrm{MP}$ absorbance spectrum used for initializing the NMF algorithm. Circles denote the samples of the in vitro macular pigment spectrum. The in vitro spectra of lutein (L) and zeaxanthin (Z) were obtained by Hammond et al. (Ref. 3). The dotted line denotes the fitted spectrum from 420 to $720 \mathrm{~nm}$ to be used for the NMF algorithm. Linear interpolation was used to fit the in vitro samples.

Thus, if after NMF applied to the above linear system we recover the spectrum of MP, i.e., $\mathbf{S}_{\mathrm{MP}}$, as one of the dominant spectral sources, then at every pixel and for every wavelength $\lambda$ we have the decomposition

$$
2 \times \mathrm{OD}_{\mathrm{MP}}(\lambda)=\mathbf{A}_{\mathrm{MP}} \times \mathbf{S}_{\mathrm{MP}}(\lambda),
$$

where $\mathbf{S}_{\mathrm{MP}}$ is the recovered spectrum and $\mathbf{A}_{\mathrm{MP}}$ is its abundance. It is customary to report the optical density at the absorbance peak, $\lambda=458 \mathrm{~nm}$, which is the target density mapped. In this study, we reported the $\mathrm{OD}_{\mathrm{MP}}$ at $\lambda=456 \mathrm{~nm}$ due to spectral resolution of $\mathbf{X}$ (i.e., 420 to $720 \mathrm{~nm}$ in $4 \mathrm{~nm}$ steps). In our case, we alternatively performed NMF on the data model

$$
\log _{10}(R)=\log _{10}\left(R_{\mathrm{SC}}\right)-2 \times \mathrm{OD}_{\mathrm{MP}}-2 \times \mathrm{OD}_{\mathrm{SubT}}
$$

and later performed the sign change to reflect the optical density of MP. The reason why a sign flip is required is due to the cost function of the NMF objective. Thus, at every pixel we mapped the optical density of MP as

$$
\operatorname{OD}_{\mathrm{MP}}(\lambda=456)=-0.5 \times \mathbf{A}_{\mathrm{MP}} \times \mathbf{S}_{\mathrm{MP}}(\lambda) .
$$

Note that although $\mathbf{S}_{\mathrm{MP}}$ may be constrained and normalized, say with value $=1$ at $\lambda=456 \mathrm{~nm}$, it does not affect the recovery of $\mathrm{OD}_{\mathrm{MP}}$ because the abundance $\mathrm{A}_{\mathrm{MP}}$ is automatically scaled so that the product gives the actual data. We note, that besides reporting $\mathrm{OD}_{\mathrm{MP}}$ at $456 \mathrm{~nm}$, the MP signature and abundance that NMF returns has a much richer spatio-spectral representation than is possible with other methods.

We performed the following normalization and initialization scheme. First, we divided each spectral band of the cube by its standard deviation to account for reflectance drifts along the spectrum. After normalization, we log-transformed the cube as just described and enforced positivity constraints on $\mathbf{X}$ to run NMF on the post-processed data. An example of $\mathbf{X}$ can be seen in Fig. 3.

The number of latent sources in the hyperspectral cube, i.e., the optimum rank of the matrix $\mathbf{S}$ of spectral signatures for recovery of the MP spectrum, was experimentally determined to be 4 . For other ranks (e.g., 3 or 5) the decomposition of the macular pigment spectra showed redundancies, i.e., similar spectral profiles. In each case, we initialized one source as $\mathbf{S}_{\mathrm{MP}}$, the in vitro absorbance prior (Fig. 4), where all other sources and abundance images were randomly initialized. However, $\mathbf{S}_{\mathrm{MP}}$ was allowed to vary during the NMF algorithm to obtain the best fit and most realistic spectrum. The obtained spatio-spectral signatures of the MP were assessed and analyzed by two retinal specialists (RTS, AF).

\section{Results}

\subsection{Dataset Description}

Figure 1 shows color fundus images of six normal eyes (N1 to N6). The resolution of the color fundus RGB images was 1052 $\times 914$. Figure 3 shows the post-processed hyperspectral cubes of a healthy subject (N6).

\subsection{Non-Negative Matrix Factorization Approach}

Table 1 summarizes the employed configuration of NMF and reports the number of iterations used, the reconstruction error in terms of the root-mean-squared (RMS) error, and other convergence specific results. The RMS error is defined as the square root of the mean difference between the data $\mathbf{X}$ and the factor 
Table 1 Reconstruction error for healthy subjects (N1 to N6). ID denotes an identifier key for a hyperspectral cube or its corresponding color fundus image, $\mathrm{N}$ is the number of iterations of the NMF algorithm, RMS is the root-mean-square residual, and D is the maximal change of either factor $A$ or $S$.

\begin{tabular}{lccc}
\hline \multicolumn{3}{c}{ Normals } \\
\hline ID & $N$ & RMS & $D$ \\
\hline N1 & 872 & 0.021399 & 0.00009994 \\
N2 & 1000 & 0.024398 & 0.00037733 \\
N3 & 1000 & 0.025532 & 0.00202895 \\
N4 & 1000 & 0.018980 & 0.00013366 \\
N5 & 1000 & 0.019192 & 0.00095528 \\
N6 & 1000 & 0.021270 & 0.00068574 \\
\hline
\end{tabular}

model AS. We used a rank $r=4$ decomposition to search for the known MP absorbance spectrum. Thus, if $\mathbf{X}$ is the hyperspectral data set, NMF found a decomposition AS as an approximation to $\mathbf{X}$, where $\mathbf{S}$ is the matrix of rank 4 of spectral signatures and $\mathbf{A}$ is the matrix of abundances. The factors $\mathbf{A}$ and $\mathbf{S}$ are chosen to minimize the RMS between $\mathbf{X}$ and AS, as previously proposed. ${ }^{44}$ For all datasets in these experiments, 1000 iterations of the algorithm resulted in a reconstruction error range of approximately 1 to $4 \%$ at each pixel.

\subsection{Recovered Macular Pigment Spectra and Optical Densities}

Figure 5 shows the recovered MP spectra. In all six eyes, the recovered spectra show the two nearby peaks in the blue characteristic of both $\mathrm{L}$ and $\mathrm{Z}$, the two carotenoids that constitute MP. ${ }^{3}$ We also found that fundus pigmentation did not have an impact on the performance of this approach, with $\mathrm{OD}_{\mathrm{MP}}$ recovered in blond (N3) as well as highly pigmented fundi (N1, N2, and N6). The corresponding $\mathrm{OD}_{\mathrm{MP}}$ maps are shown in Fig. 6. $\mathrm{OD}_{\mathrm{MP}}$ values ranged from 0.08 to 0.22 (mean $0.15 \pm 0.05$, Table 2). Figure 7 shows a larger view of the $\mathrm{OD}_{\mathrm{MP}}$ map for subject $\mathrm{N} 1$ and corresponding MP density profile plot along the horizontal meridian.

\section{Discussion}

The hyperspectral tool we have utilized herein is an integration of highly novel imaging technology and the blind source separation method of NMF. The high spectral resolution $(4 \mathrm{~nm})$ of the CTIS multiplexing device coupled with the ability of NMF to precisely detect a spectral signal and its abundance, have together achieved a detailed demonstration of the two absorbance peaks of MP in normal eyes in vivo. To our knowledge, this is the first demonstration of the bifid absorbance spectrum of MP in vivo. Other researchers have attempted to fit their recovered spectral signatures to known MP spectra, ${ }^{34}$ which is rather different to our approach. The flexibility allowed by having multiple spectral points in the region of interest (21 measurements
Table 2 Macular pigment optical density $\left(\mathrm{OD}_{\mathrm{MP}}\right)$ for healthy subjects (normals). ID denotes an identifier key for a hyperspectral cube or its corresponding color fundus image, Max is the peak optical density value within a two degree annuli centered at the fovea, Mean is the average optical density within the same region as Max, and SD is its standard deviation.

\begin{tabular}{lccc}
\hline & \multicolumn{3}{c}{ Normals } \\
\hline ID & Max & Mean & SD \\
\hline N1 & 0.14504 & 0.14243 & 0.00531 \\
N2 & 0.22397 & 0.22117 & 0.00987 \\
N3 & 0.10200 & 0.07576 & 0.01001 \\
N4 & 0.16015 & 0.13787 & 0.01581 \\
N5 & 0.18881 & 0.16846 & 0.01847 \\
N6 & 0.17816 & 0.17613 & 0.00531 \\
\hline
\end{tabular}

at each pixel in the region from 420 to $500 \mathrm{~nm}$ ), and the use of MP signature only to initialize NMF search, give this approach the distinct advantage of reaching MP spectral signature based on individual eye's spatial/spectral data encoded in the hyperspectral cube. The accuracy of this method is demonstrated by the highly resolved recovered spectral signal in normal subjects. Moreover, the method is versatile in its ability to recover the spectra of known or unknown reflectors and absorbers. While in this project we used the known in vitro spectrum of MP to guide the recovery of its abundance, we have previously utilized the same blind source separation in a completely unsupervised fashion to extract spectral signatures that consistently co-localized with drusen. ${ }^{28,29}$ Suggesting this approach can be used to achieve important tasks in the study of the normal and diseased fundus.

Advantages of this approach include its relative freedom from dependence on visual acuity or fixation, which may be highly valuable when evaluating patients with unstable and/or eccentric fixation. This is one of the main limitations of psychophysical approaches to MP assessments, such as HFP and customized HFP. Another limitation of the psychophysical approach relates to its basic assumption that $\mathrm{OD}_{\mathrm{MP}}$ is zero outside the central $5 \mathrm{deg}$, which leads to its systematic underestimation of $\mathrm{OD}_{\mathrm{MP}}$ when compared with other approaches. ${ }^{36,46-48}$ It was previously shown that MP can still be present at low levels at $1 \mathrm{~mm}$ eccentricity $(\approx 4 \mathrm{deg})$ in primate eyes ${ }^{49}$ and out to $8 \mathrm{deg}$ in human donor eyes. ${ }^{50} \mathrm{~A}$ residual signal in our data at more eccentric locations might be attributed to this low level. A further advantage of the snapshot hyperspectral method is the capability to map a complete spatial profile of MP. Other hyperspectral methods, although they may use careful fits to models of fundus reflectance, only acquire MP density at a single point, for example, a measure of the averaged density in the central $1.9 \mathrm{deg} .{ }^{51}$ The snapshot method acquires a full three-dimensional spectral-spatial cube, which is simultaneously analyzed to measure MP density at every point of a $4 \mathrm{~mm}$ square field at a resolution of $22 \mu \mathrm{m}$.

The limitations of this approach include the inherent restrictions of measuring MP with reflectance. Delori et al. have 
N1

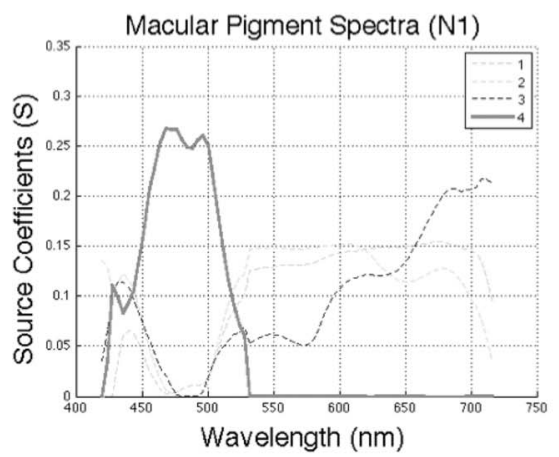

N4



N2

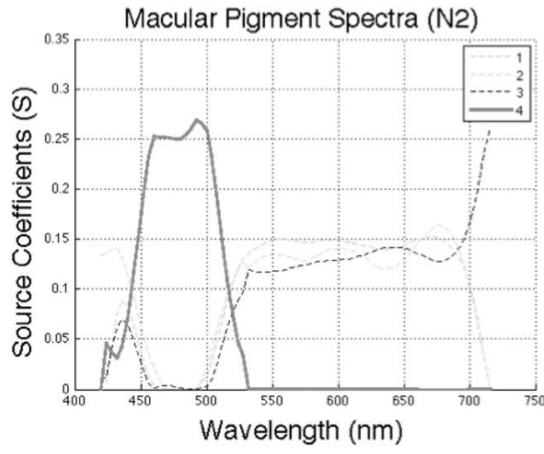

N5

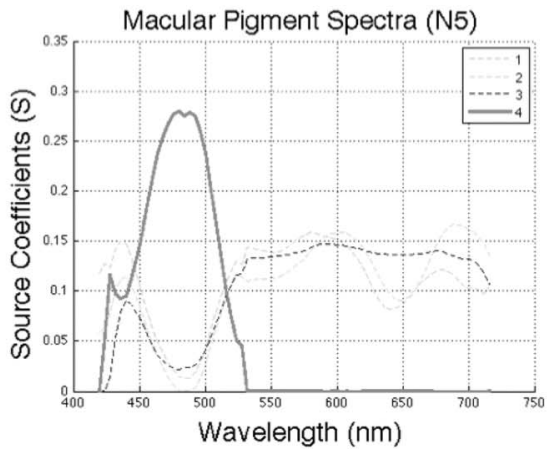

N3

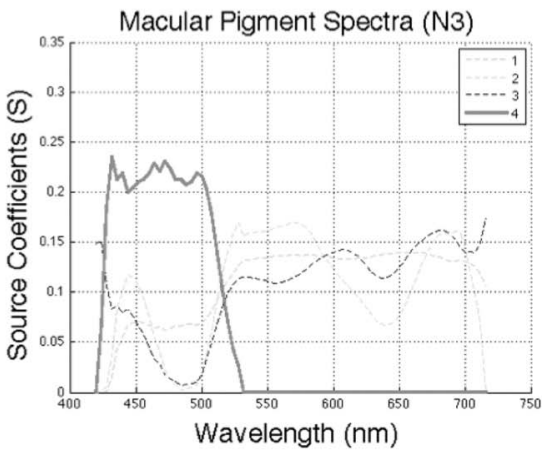

N6

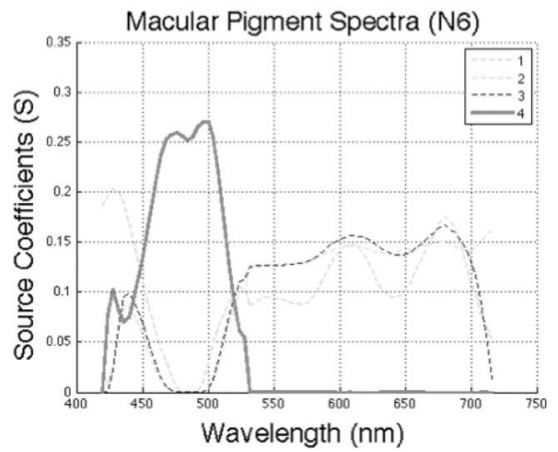

Fig. 5 Recovered spectra for normal subjects. The four spectra recovered by NMF for each of the six healthy subjects (N1-N6). The fourth spectrum denotes the MP spectrum (solid line). The two peaks between 450 and $500 \mathrm{~nm}$ are the classic bifid spectrum previously reported (Ref. 3) in vitro. The secondary peaks at $425 \mathrm{~nm}(\mathrm{~N} 1, \mathrm{~N} 2, \mathrm{~N} 4, \mathrm{~N} 5$, and $\mathrm{N} 6)$ have also been reported in vitro.

provided a detailed analysis showing that while MP measurements are highly correlated among three different approaches, reflectance values are much lower than other approaches, and offered several explanations, including the presence of anterior reflectors in the retina and lens scatter. ${ }^{36}$ The low $\mathrm{OD}_{\mathrm{MP}}$ values obtained in our subjects are consistent with these previous findings obtained by reflectometry. ${ }^{36}$ Methods to correct these errors include baseline spectral measures of lens scatter ${ }^{36}$ and model-based estimates of (anterior reflectance) foveal reflectance using four spectral lines. ${ }^{45}$ These corrections could be implemented in future work. Another limitation may be related to the possible chromatic aberrations and defocus inherent in the spectral range at issue (420 to 500), although our dataset showed essentially uniform image quality in this range (Fig. 3). The optical density of the individual eye in principle should not confound the NMF hyperspectral method for $\mathrm{OD}_{\mathrm{MP}}$, because it is contained in $\mathrm{OD}_{\mathrm{SubT}}$, the separate term for other absorbers. Absorption by incompletely bleached cone photoreceptor pigment could also influence the hyperspectral data, but again, in principle, these effects should be separated from the MP by the NMF method, which specifically isolates the contribution from the MP spectrum. In fact, there could be imperfect unmixing of these signals. In the case of cones, the peak absorbances of the short and medium wavelength cones are centered about $50 \mathrm{~nm}$ on either side of the peaks of the MP profile, ${ }^{52}$ hence, they would have only a relatively small effect, similar to that of photoreceptor absorbance on HFP depending on differential absorption of blue and green light. Finally, the internal limiting membrane reflectance signal is apparent in the clinical photos of the younger normal subjects N2 and N6, and to a lesser extent
$\mathrm{N} 1$

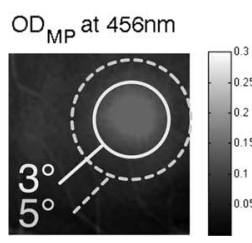

N2

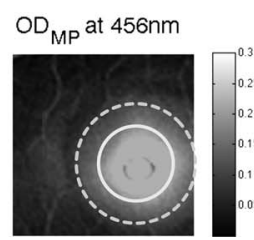

N3

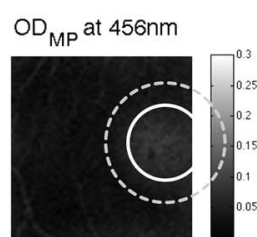

N4

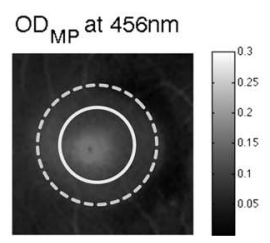

N5

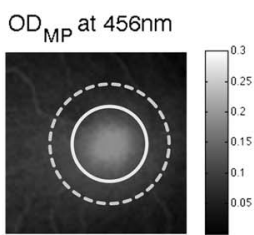

N6

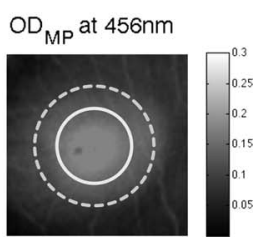

Fig. 6 Hyperspectral macular pigment optical density $\left(\mathrm{OD}_{\mathrm{MP}}\right)$ maps. Results of NMF applied to the corresponding hyperspectral data cubes, resulting in abundance maps (not shown) that have been converted to OD MP maps at $456 \mathrm{~nm}$ (grayscale coded). In the six healthy subjects (N1 to N6), the peak $\mathrm{OD}_{\mathrm{MP}}$ ranged from 0.08 to 0.22 (Table 2) and became negligible at 4 to 6 deg eccentricity, consistent with the known distribution of MP (Ref. 53). 

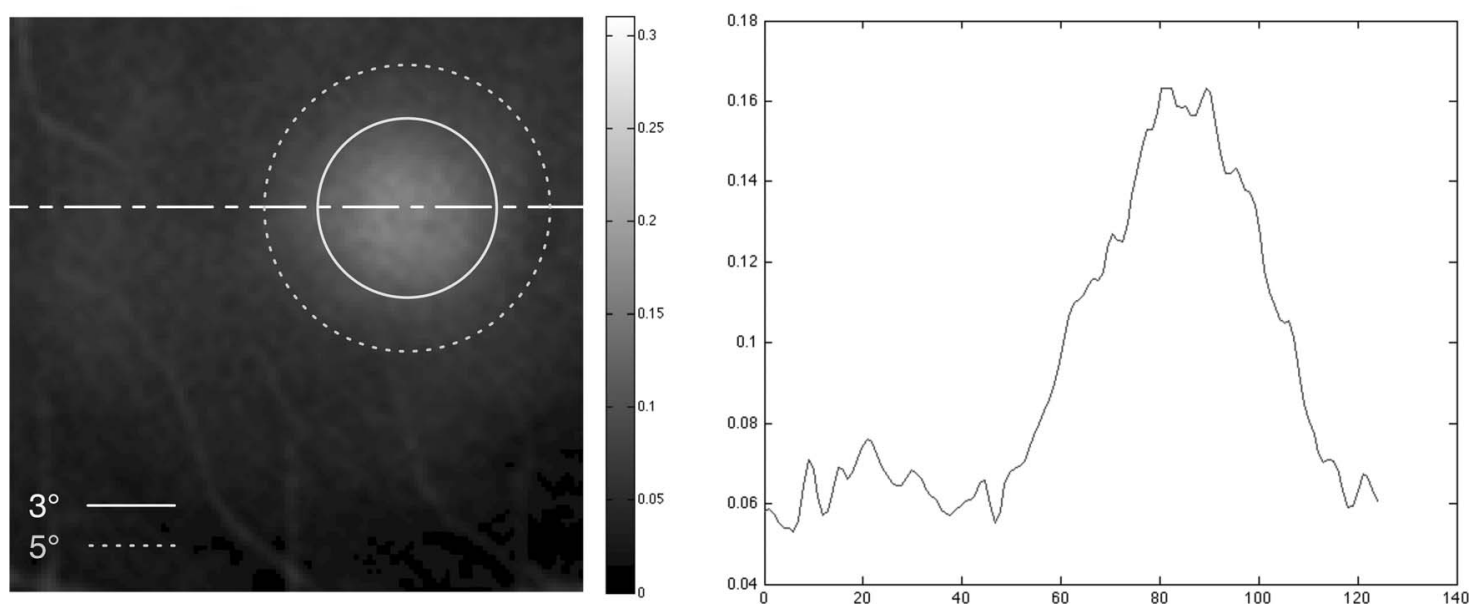

Fig. 7 Detailed view of a hyperspectral macular pigment optical density (ODMP) map for subject $\mathrm{N} 1$ and macular pigment density profile plot. A larger view of the $\mathrm{OD}_{\mathrm{MP}}$ map at $456 \mathrm{~nm}$ for subject $\mathrm{N} 1$ is shown (left) and a density profile plot along the horizontal meridian is given (right), corresponding to the horizontal white dotted line.

in other images, overall, the effect of scattering due the internal limiting membrane is small..$^{51}$

It is important to note that approaches using differential reflectance or AF images to calculate MP density maps rely on major assumptions. For reflectance, the entire difference in reflectance between a single blue and green wavelength is attributed to MP. The NMF method makes no such assumptions and instead uses the full spectral information in the 27 bands between 420 and $530 \mathrm{~nm}$ to fit the reflectance data to a detailed prior model of the MP spectrum. Similarly, the AF method makes several assumptions. First, the difference between AF intensity centrally and peripherally is entirely due to the absorbance by MP of blue light. Second, the relative spectral energy of lipofuscin fluorescence is constant across the central retina and, last, that MP density is zero at a peripheral point, another potential source of error for all comparison methods. Again, the strength of the NMF method is that it directly searches for the absorber of interest based on its own signature and specifically separates the distribution of this signature from the other components of the data. No assumptions are made or required about the distribution of other absorbers and reflectors.

There are potential advantages and disadvantages to the general NMF method and specifically to the use of an initialized choice of spectral basis vector. The power of NMF lies in its ability to extract meaningful spectra from data without prior knowledge. For example, this would apply to the reflectance spectra of drusen, which are unknown. In such cases, the initial basis vectors are specified in number only (the rank of the decomposition) and otherwise, the initial spectra can be virtually random. This approach is the broadest but with no guarantee that meaningful spectra will be recovered. However, NMF is also used in the context where a specific approximate spectral signal is known to be present in the data. Thus, the power of the NMF approach with prior knowledge is that, by specifying one initial basis vector for the in vivo spectrum of MP, the algorithm is already "close" to a solution. During the solution, the algorithm modifies the initial basis vector and calculates its spatial distribution as necessary to find the best objective fit to real closely-spaced spatio-spectral data. Recovery of both a physiologically reasonable MP spectrum and density profile in turn, provides substantial evidence that the NMF method has effectively identified the MP and separated it from other ocular absorbers, even though no other absorbers were explicitly modeled.

Realistically, no spectral separation will be perfect. Some of the abundance images for MP in normal eyes show signal in the retinal vessels, suggesting that hemoglobin absorbance has not been completely separated from the MP spectrum. Indeed, because we did not explicitly include hemoglobin absorbance spectra in our other basis vectors, a slight hemoglobin admixture would be expected. Moreover, the MP distributions in other published methods highlight the vessels, ${ }^{20,23,27}$ suggesting that a complete separation of hemoglobin and MP has not yet been achieved. A simple future solution would be an initial segmentation of the vessels, either manually or by automated techniques. A more sophisticated solution, directly amenable to NMF, would be to search for hemoglobin basis vectors.

A future goal will be to separately measure $\mathrm{L}$ and $\mathrm{Z}$. Their spectra are similar, with $\mathrm{Z}$ shifted to the red by $10 \mathrm{~nm}$. We believe that this should be possible to achieve with our method, as suggested by the fact that separate $\mathrm{L}$ and $\mathrm{Z}$ optical densities have been measured using fundus reflectometry with $5.8 \mathrm{~nm}$ resolution in vivo ${ }^{32}$ (although the actual spectra were not recovered in this experiment). Our method has shown the ability to resolve peaks $10 \mathrm{~nm}$ apart, hence, it should also be able to recover the $\mathrm{L}$ and $\mathrm{Z}$ spectra.

In summary, we have shown that the combination of a novel hyperspectral imaging tool with advanced mathematical analysis can, to the best of our knowledge, for the first time, recover detailed spectral absorption curves for MP in vivo that correspond to physically realistic retinal distributions. The calculated optical densities are consistent with other reflectometry methods. With regard to MP specifically, the ease, speed, and precision of MP density acquisition suggests that further effort with known methods is warranted to correct for error sources such as lens scatter and pre-retinal reflectors. More generally, success with MP suggests that this hyperspectral method will prove effective in recovering known and unknown spectra and densities of 
important ocular reflectors and absorbers. Among these, which should be investigated, are drusen in age-related macular degeneration, whose complex biochemical composition is critical to understanding the disease process.

\section{Acknowledgments}

The authors would like to thank Jennifer Dalberth for editorial assistance, the New York Community Trust (RTS), NEI R01 EY015520 (RTS), and R01 EY021470 (RTS, AAF) for Research support, and Reichert, Inc. (AAF) for CR: Grant support.

\section{References}

1. J. J. Nussbaum, R. C. Pruett, and F. C. Delori, "Historic perspectives. Macular yellow pigment. The first 200 years," Retina 1(4), 296-310 (1981).

2. K. Kirschfeld, "Carotenoid pigments: their possible role in protecting against photooxidation in eyes and photoreceptor cells,"Proc. R. Soc. London, Ser. B 216(1202), 71-85 (1982).

3. B. R. Hammond, B. R. Wooten, and B. Smollon, "Assessment of the validity of in vivo methods of measuring human macular pigment optical density," Optom. Vision Sci. 82(5), 387-404 (2005).

4. F. Khachik, P. S. Bernstein, and D. L. Garland, "Identification of lutein and zeaxanthin oxidation products in human and monkey retinas," Invest. Ophthalmol. Visual Sci. 38(9), 1802-1811 (1997).

5. S. Beatty, I. J. Murray, D. B. Henson, D. Carden, H. H. Koh, and M. E. Boulton, "Macular pigment and risk for age-related macular degeneration in subjects from a Northern European population," Invest. Ophthalmol. Visual Sci. 42(2), 439-446 (2001).

6. J. M. Nolan, J. Stack, O. O. Donovan, E. Loane, and S. Beatty, "Risk factors for age-related maculopathy are associated with a relative lack of macular pigment," Exp. Eye Res. 84(1), 61-74 (2007).

7. T. T. Berendschot, J. J. Willemse-Assink, M. Bastiaanse, P. T. de Jong, and D. van Norren, "Macular pigment and melanin in age-related maculopathy in a general population," Invest. Ophthalmol. Visual Sci. 43(6), 1928-1932 (2002).

8. M. Dietzel, M. Zeimer, B. Heimes, B. Claes, D. Pauleikhoff, and H. W. Hense, "Determinants of macular pigment optical density and its relation to age-related maculopathy - results from the muenster aging and retina study (MARS)," Invest. Ophthalmol. Visual Sci. 52(6), 3452-3457 (2011).

9. S. Beatty, J. Nolan, H. Kavanagh, and O. O'Donovan, "Macular pigment optical density and its relationship with serum and dietary levels of lutein and zeaxanthin," Arch. Biochem. Biophys. 430(1), 70-76 (2004).

10. E. Loane, C. Kelliher, S. Beatty, and J. M. Nolan, "The rationale and evidence base for a protective role of macular pigment in age-related maculopathy," Br. J. Ophthalmol. 92(9), 1163-1168 (2008).

11. B. R. Hammond and K. Fuld, "Interocular differences in macular pigment density," Invest. Ophthalmol. Visual Sci. 33(2), 350-355 (1992).

12. B. R. Hammond, B. R. Wooten, and D. M. Snodderly, "Individual variations in the spatial profile of human macular pigment," J. Opt. Soc. Am. A Opt. Image Sci. Vis. 14(6), 1187-1196 (1997).

13. P. L. Pease, A. J. Adams, and E. Nuccio, "Optical-density of human macular pigment," Vision Res. 27(5), 705-710 (1987).

14. R. A. Bone and J. T. Landrum, "Heterochromatic flicker photometry," Arch. Biochem. Biophys. 430(2), 137-142 (2004).

15. D. M. Snodderly, J. A. Mares, B. R. Wooten, L. Oxton, M. Gruber, T. Ficek, and C. M. P. S. Grp, "Macular pigment measurement by heterochromatic flicker photometry in older subjects: The carotenoids and age-related eye disease study," Invest. Ophthalmol. Visual Sci. 45(2), 531-538 (2004).

16. B. R. Wooten, B. R. Hammond, R. I. Land, and D. M. Snodderly, "A practical method for measuring macular pigment optical density," Invest. Ophthalmol. Visual Sci. 40(11), 2481-2489 (1999).

17. J. D. Moreland, A. G. Robson, and J. J. Kulikowski, "Macular pigment assessment using a colour monitor," Color Res. Appl. 26, S261-S263 (2001).
18. J. D. Moreland, "Macular pigment assessment by motion photometry," Arch. Biochem. Biophys. 430(2), 143-148 (2004).

19. G. S. Brindley and E. N. Willmer, "The reflexion of light from the macular and peripheral fundus oculi in man," J. Physiol. 116(3), 350 356 (1952).

20. T. Berendschot, P. J. DeLint, and D. van Norren, "Fundus reflectance - historical and present ideas," Prog. Retin. Eye Res. 22(2), 171-200 (2003).

21. F. C. Delori and K. P. Pflibsen, "Spectral reflectance of the human ocular fundus," Appl. Opt. 28(6), 1061-1077 (1989).

22. F. C. Delori, "Spectrophotometer for noninvasive measurement of intrinsic fluorescence and reflectance of the ocular fundus," Appl. Opt. 33(31), 7439-7452 (1994).

23. F. C. Delori, "Autofluorescence method to measure macular pigment optical densities fluorometry and autofluorescence imaging," Arch. Biochem. Biophys. 430(2), 156-162 (2004).

24. A. G. Robson, J. D. Moreland, D. Pauleikhoff, T. Morrissey, G. E. Holder, F. W. Fitzke, A. C. Bird, and F. J. van Kuijk, "Macular pigment density and distribution: comparison of fundus autofluorescence with minimum motion photometry," Vision Res. 43(16), 1765-1775 (2003).

25. P. S. Bernstein, D. Y. Zhao, S. W. Wintch, I. V. Ermakov, R. W. McClane, and W. Gellermann, "Resonance Raman measurement of macular carotenoids in normal subjects and in age-related macular degeneration patients," Ophthalmology 109(10), 1780-1787 (2002).

26. P. S. Bernstein, M. D. Yoshida, N. B. Katz, R. W. McClane, and W. Gellermann, "Raman detection of macular carotenoid pigments in intact human retina," Invest. Ophthalmol. Visual Sci.39(11), 2003-2011 (1998).

27. P. S. Bernstein, F. C. Delori, S. Richer, F. J. M. van Kuijk, and A. J. Wenzel, "The value of measurement of macular carotenoid pigment optical densities and distributions in age-related macular degeneration and other retinal disorders," Vision Res. 50(7), 716-728 (2010).

28. N. Lee, J. Wielaard, A. A. Fawzi, P. Sajda, A. F. Laine, G. Martin, M. Humayun, and R. T. Smith, "In vivo snapshot hyperspectral image analysis of age-related macular degeneration," in Proc. of the 32nd Annual International Conference of the IEEE Engineering in Medicine and Biology Society (EMBC), Buenos Aires, Argentina (2010).

29. R. T. Smith, P. Sajda, A. A. Fawzi, A. Kashani, G. Bearman, D. Wilson, B. Johnson, G. Martin, and M. Humayun, "Drusen spectral signatures via unsupervised spectral unmixing of snapshot hyperspectral images," in ARVO-ISIE (2009).

30. V. Nourrit, J. Denniss, M. M. Muqit, I. Schiessl, C. Fenerty, P. E. Stanga, and D. B. Henson, "High-resolution hyperspectral imaging of the retina with a modified fundus camera," J. Fr. Ophthalmol. 33(10), 686-692 (2010).

31. D. Bert, R. Steven, A. Michael, C. N. Sheila, E. S. Barriga, and S. Peter, Identification of spectral phenotypes in age-related macular degeneration patients, M. Fabrice, G. S. Per, H. Arthur, E. S. Bruce, and B. Michael, Eds., p. 64261I, SPIE (2007).

32. J. van de Kraats, M. J. Kanis, S. W. Genders, and D. van Norren, "Lutein and zeaxanthin measured separately in the living human retina with fundus reflectometry," Invest. Ophthalmol. Visual Sci. 49(12), 55685573 (2008).

33. R. A. Bone, B. Brener, and J. C. Gibert, "Macular pigment, photopigments, and melanin: distributions in young subjects determined by fourwavelength reflectometry," Vision Res. 47(26), 3259-3268 (2007).

34. P. E. Kilbride, K. R. Alexander, M. Fishman, and G. A. Fishman, "Human macular pigment assessed by imaging fundus reflectometry," Vision Res. 29(6), 663-674 (1989).

35. S. F. Chen, Y. Chang, and J. C. Wu, "The spatial distribution of macular pigment in humans," Curr. Eye Res. 23(6), 422-434 (2001).

36. F. C. Delori, D. G. Goger, B. R. Hammond, D. M. Snodderly, and S. A. Burns, "Macular pigment density measured by autofluorescence spectrometry: comparison with reflectometry and heterochromatic flicker photometry," J. Opt. Soc. Am. A Opt. Image Sci. Vis. 18(6), 1212-1230 (2001).

37. A. E. Elsner, S. A. Burns, E. Beausencourt, and J. J. Weiter, "Foveal cone photopigment distribution: small alterations associated with macular pigment distribution," Invest. Ophthalmol. Visual Sci. 39(12), 2394 2404 (1998). 
38. J. Z. Xie, W. Johnson, N. Walsh, D. Wilson, G. Bearman, and M. Humayun, "Snap-shot human retinal oximetry," Invest. Ophthalmol. Visual Sci. 47 (ARVO E-Abstract), 3306 (2006).

39. W. R. Johnson, D. W. Wilson, W. Fink, M. Humayun, and G. Bearman, "Snapshot hyperspectral imaging in ophthalmology," J. Biomed. Opt. 12(1), 014036 (2007).

40. D. Schweitzer, M. Hammer, K. J. Thamm, E. Konigsdorffer, and J. Strobel, "In vivo measurement of the oxygen saturation of retinal vessels in healthy volunteers," IEEE Trans. Biomed. Eng. 46(12), 14541465 (1999).

41. D. D. Lee and H. S. Seung, "Learning the parts of objects by nonnegative matrix factorization," Nature 401(6755), 788-791 (1999).

42. P. Sadja, S. Du, and L. Parra, "Recovery of constituent spectra using non-negative matrix factorization," Proc. SPIE 5207, 321-331 (2003).

43. P. Sajda, "Recovery of constituent spectra in 3D chemical shift imaging using non-negative matrix factorization," in 4th Int. Symposium on Independent Component Analysis and Blind Signal Separation, Nara, Japan (2003).

44. P. Sajda, S. Y. Du, T. R. Brown, R. Stoyanova, D. C. Shungu, X. L. Mao, and L. C. Parra, "Nonnegative matrix factorization for rapid recovery of constituent spectra in magnetic resonance chemical shift imaging of the brain," IEEE Trans. Biomed. Eng. 23(12), 1453-1465 (2004).

45. J. van de Kraats, T. Berendschot, and D. vanNorren, "The pathways of light measured in fundus reflectometry," Vision Res. 36(15), 2229-2247 (1996).
46. S. Beatty, F. van Kuijk, and U. Chakravarthy, "Macular pigment and age-related macular degeneration: longitudinal data and better techniques of measurement are needed," Invest. Ophthalmol. Visual Sci. 49(3), 843-845 (2008).

47. T. T. Berendschot and D. van Norren, "On the age dependency of the macular pigment optical density," Exp. Eye Res. 81(5), 602-609 (2005).

48. R. L. P. van der Veen, T. Berendschot, M. Makridaki, F. Hendrikse, D. Carden, and I. J. Murray, "Correspondence between retinal reflectometry and a flicker-based technique in the measurement of macular pigment spatial profiles," J. Biomed. Opt. 14(6), 064046 (2009).

49. D. M. Snodderly, J. D. Auran, and F. C. Delori, "The macular pigment. II. Spatial distribution in primate retinas," Invest. Ophthalmol. Visual Sci. 25(6), 674-685 (1984).

50. R. A. Bone, J. T. Landrum, S. T. Mayne, C. M. Gomez, S. E. Tibor, and E. E. Twaroska, "Macular pigment in donor eyes with and without AMD: a case-control study," Invest. Ophthalmol. Visual Sci. 42(1), 235-240 (2001).

51. T. Berendschot and D. van Norren, "Objective determination of the macular pigment optical density using fundus reflectance spectroscopy," Arch. Biochem. Biophys. 430(2), 149-155 (2004).

52. S. L. Merbs and J. Nathans, "Absorption spectra of human cone pigments," Nature 356(6368), 433-435 (1992).

53. D. M. Snodderly, P. K. Brown, F. C. Delori, and J. D. Auran, "The macular pigment. I. Absorbance spectra, localization, and discrimination from other yellow pigments in primate retinas," Invest. Ophthalmol. Visual Sci. 25(6), 660-673 (1984). 